\title{
Factors Influencing Uptake of Changes to Clinical Preventive Guidelines
}

\author{
Vivian Jiang, MD, E. Marshall Brooks, PhD, Sebastian T. Tong, MD, MPH, \\ John Heintzman, MD, and Alex H. Krist, MD, MPH
}

Background: Despite widespread recognition that adherence to clinical preventive guidelines improves patient outcomes, clinicians struggle to implement guideline changes in a timely manner. Multiple factors influence guideline adoption and effective implementation. However, few studies evaluate their collective and inter-related effects. This qualitative study provides a comprehensive picture of the interplay between multiple factors on uptake of new or changed preventive guidelines.

Methods: Semistructured interviews conducted in 2018 with a diverse sample of clinicians and practice leaders sought to understand patient, clinician, practice, health system, environment, and guideline factors of influence. An immersion-crystallization approach was used to identify emergent themes.

Results: Interviewees expressed motivation to adhere to guidelines but also valued sharing decisions with patients. Personal biases and fears affected both clinician and patient guideline adoption. Practices facilitated implementation through workflow optimization and encouraging a culture of evidence-based practice while a key health system function was to maintain electronic health record alerts. More traditional environmental factors, such as insurance coverage or transportation, were less of a barrier to guideline adoption and implementation than the influence of media and specialists. Various specific guideline characteristics also affected ease of adoption and implementation. Different settings expressed greater health system, practice, or clinician-centric approaches to guideline implementation.

Conclusions: Guideline uptake is influenced by a complex interplay of multiple levels of factors including the patient, clinician, practice, health system, environment, and guideline levels. Comprehensively understanding all levels of influence for each specific clinical setting may help to determine the optimal intervention(s) for improving uptake of evidence-based guidelines. ( $\mathrm{J}$ Am Board Fam Med 2020;33:271-278.)

Keywords: Evidence-Based Medicine, Implementation Science, Preventive Medicine, Qualitative Research

\section{Introduction}

Organizations like the US Preventive Services Task Force and Advisory Committee on Immunization Practices translate complex scientific evidence into clinical preventive guidelines. These guidelines are

This article was externally peer reviewed.

Submitted 21 April 2019; revised 9 August 2019; accepted 11 August 2019.

From the Department of Family Medicine and Population Health, Virginia Commonwealth University, Richmond, VA (VJ, EMB, AHK); Department of Family Medicine, Oregon Health Sciences University, Portland, OR (JH); Oregon Community Health Information Network (OCHIN), Portland OR (JH).

Funding: This project was funded by the Agency for Healthcare Research and Quality (R03 HS25032-01), National Cancer Institute (R01 CA168795-01), and National Center for Advancing Translational Sciences (UL1TR002649). The content is solely the responsibility of regularly updated with the most up-to-date evidence. ${ }^{1,2}$ Despite wide recognition that adherence to evidence-based guidelines improves outcomes, clinicians struggle to implement guidelines in a timely fashion. ${ }^{3-5}$ Meanwhile, overscreening of breast, cervical, and prostate cancer, underscreening of lung cancer, and suboptimal vaccination rates continue to put patients at harm. ${ }^{4-7}$

the authors and does not necessarily represent the official views of the Agency for Healthcare Research and Quality.

Conflicts of interest: AHK is the Vice Chair for the United States Preventive Services Task Force (USPSTF). This manuscript does not necessarily represent the views and policies of the USPSTF. The other authors have no conflicts of interest nor financial disclosures to declare.

Corresponding author: Vivian Jiang, MD, 12631 East 17th Avenue, Box F496, Aurora, CO 80045 (E-mail: Vivian. Jiang@cuanschutz.edu). 
Many factors have been shown to influence guideline uptake, such as guideline characteristics (eg, ease of implementation, guideline clarity), clinician familiarity with guidelines and evidence, and patient comorbidities or awareness of need. ${ }^{8,9}$ Interventions targeting these barriers, such as clinician and patient education or system-level changes like alerts or team-based care, have been shown effective in increasing guideline uptake. ${ }^{10}$ However, few studies provide a comprehensive evaluation of how different levels of factors collectively affect guideline uptake. ${ }^{11}$ Examining these factors as a whole and how they interact may better inform interventions. This qualitative study details clinician and practice leader perceptions on how patient, clinician, practice, health system, environment, and guideline factors impact preventive service guideline adoption and implementation.

\section{Methods}

Using a qualitative approach, this study examined factors influencing clinical guideline uptake. Interviews were conducted and analyzed between June and August 2018. This study was approved by the Virginia Commonwealth University Institutional Review Board. Interviewees were not compensated for participation.

\section{Participants}

We interviewed 15 clinicians and 9 practice leaders representing 15 diverse primary care practices in 5 states (Montana, North Carolina, Ohio, Oregon, Virginia) from 2 research networks (Virginia Ambulatory Outcomes Research Network [ACORN] and Oregon Community Health Information Network [OCHIN]). We solicited interviewees by emailing and calling practices, purposely selecting practices for diverse settings (eg, urban/rural) and populations served. Practices identified clinicians and leaders for participation based on involvement with ensuring guideline adoption and implementation.

\section{Interviews}

Using a semistructured interview guide (see online Appendix 1), interviewees were asked how they learn about guideline changes and what patient, clinician, practice, health system, environment, and guideline factors influence uptake. While the interview focused on guidelines generally, interviewees were prompted to think about prevention guidelines from the US Preventive Services Task Force and Advisory Committee on Immunization Practices. ${ }^{12-19}$ "Adoption" was defined as the decision to accept a guideline and "implementation" as the process to ensure delivery of the service. One interviewer (VJ) conducted all interviews by phone. Interviews were voice recorded and transcribed. Interviews were scheduled until thematic saturation was reached.

\section{Qualitative Analysis}

Two reviewers (AK and VJ) independently coded transcripts using grounded theory and an immersion-crystallization process to identify recurring themes and subthemes. ${ }^{20,21}$ Discrepancies were resolved by discussion among $\mathrm{AK}, \mathrm{VJ}$, and $\mathrm{MB}$.

\section{Results}

Interviewees included 14 physicians, 1 nurse practitioner, 1 physician practice manager, 6 nurse managers, and 2 nonclinical office managers. Interviewees had 3 to 35 years' experience and represented 5 suburban private practices, 5 federally qualified health centers, and 5 university clinics, including 13 family medicine and 2 internal medicine practices.

There was general agreement regarding the most prominent factors affecting guideline adoption and implementation (Table 1). Interviewees described personal biases and fears of negative outcomes as major factors affecting guideline adoption for both patients and clinicians. Interviewees reported that informed health-literate patients sometimes wanted more care than guidelines recommended. While most clinicians were familiar with the latest changes in evidence, several mentioned that personal beliefs or negative experiences led them to delay fully adopting new guidelines, especially if changes involved de-escalation of services. The main patient factor influencing uptake was patients' relationship with and trust in their clinician. Likewise, dedication to patient-centered care was a major clinician factor affecting how clinicians implemented guideline changes; clinicians expressed an overwhelming preference for sharing decisions with patients rather than pushing new guidelines onto patients.

Discussion of practice and health system factors centered around diffusing knowledge and standardiz- 
Table 1. Clinician and Practice Leader Perspectives on Factors Affecting Implementation of Changes in Evidence

Themes and Findings $\quad$ Example Quotes

\section{Patient Factors}

Trust and fear strongly influence patient decisions

- Patients largely rely on clinician recommendations

- Patients appreciate clinicians explaining how a guideline applies to them

- Guideline changes can foster patient distrust

- Doing less sometimes makes patients feel less cared for

- Personal experiences influence decisions

Patient education (mostly) helps guideline adoption

- Informed patients are typically more engaged and activated

- But being more engaged does not always mean ready to change

- Less engaged patients may do whatever their doctors recommend

- Misinformation can undermine evidence-based care

- But being accurately informed about the evidence does not necessarily mean a patient will want to follow the evidence

Clinician Factors

Clinicians believe in tailoring guidelines to individual patients

- Clinicians pride themselves on knowing their patients

- Clinicians like to discuss guidelines and share decisions with patients

- Clinicians may prioritize other patient needs (co-morbidities, patient beliefs, cost) over guidelines

Clinicians' personal beliefs impact guideline adoption

- Clinicians are quicker to adopt guidelines that they agree with and make sense

- Clinicians' personal healthcare experiences at times inform guideline recommendations given to patients

- De-escalating services can create fear of missing something

- Negative patient outcomes from prior misses can increase fear Practice Factors

Attention to workflow and staff roles support guideline implementation

- Integrating guidelines into clinic workflow decreases dependence on clinician memory

- Clinical support staff who work at the top of their licenses can enhance the promotion of guidelines

- Practices dedicated protected time to define workflow and roles

Practice culture shapes the adoption and implementation of evidence-based guidelines

- Practices work to continually evolve and become better

- Having a teaching mission helps build a culture of evidencebased care and keeping up-to-date with guidelines.

- Practices have regular meetings to share knowledge about care

- Clinicians and staff who participate in committees to define health system policies carry knowledge back to their practices

- Practices view quality improvement as a continual process

Health System Factors

Maintaining EHR functionality is the main way health systems promote guideline adoption and implementation

- EHR alerts are useful for reinforcing and reminding clinicians about guidelines

- EHR templates and standing orders can further incorporate rooming staff into workflow when implementing a new guideline

- Patient portals can help communicate guidelines to patients

- There are noticeable gaps in EHR alerts and functionality
"I think most patients just want you to make a recommendation. Like prostate cancer, I support informed decision-making and they say, "just tell me what to do." (Clinician)

"I always knew that doing yearly paps was not a good idea...A lot of that stuff, especially when it's less services, I'm only doing because patients are so used to it." (Clinician)

"Quite a few folks are leery about statins. The've seen ads on TV saying there are potential side effects. 'I know my Aunt Suzi had problems and I'm not going to do that to myself."” (Clinician)

"I think our population is pretty well educated but, by the same token, they're also creatures of habit. Nobody likes change; everyone resists change." (Practice Leader)

"Sometimes people educate themselves and are all for following guidelines, other folks have educated themselves and have determined they are pretty hesitant." (Clinician)

"I'm a big believer in kind of the mutual decision; not just me telling them what to do, and realistically if they don't believe what I'm saying they won't do it anyway." (Clinician)

"We had a patient who died of cervical cancer and she had had a Pap smear six months before that was normal ... you know if you're counting on a test to give you a five year pass, that's a long time." (Clinician)

"I always knew that doing yearly Paps was not a good idea. I was sort of waiting for that to happen."

(Clinician)"Mammography. .. I tell them that I personally am experimenting on myself with every other year but I let them decide." (Clinician)

"When we weren't getting workflows going for new guidelines, some providers were doing it and some weren't and it was sort of left up to memory... sometimes you leave too much up to the individual provider and there's just too much to keep in your head." (Clinician)

"We're trying to have the nurses [sic] be the frontline more and more. . They are protecting the provider's time..." (Clinician)

"Once a month [we hold] a provider meeting where we do peer review...[and discuss] case studies or interesting fun facts." (Practice Leader)

"We participate in a couple of committees. ..then there are practice councils that our nurses attend. ..I would say updates regarding guidelines come through all those different avenues" (Practice Leader)

"The quality tab has been very helpful because of the prompts that it offers you for things that you might have otherwise forgotten." (Clinician)

"We are guided into following or keeping up-to-date on guidelines because it's entered in the medical record. . .it flags us that somebody is due for something." (Clinician)

"If it's something that is brand-new, like when Shingrix came out, we take it to the quality [committee] for approval, and then we send an email out to the clinical staff, the clerical staff, and the providers." (Practice Leader) 
Health systems standardize guideline adoption and implementation processes

- Health system committees often review and make recommendations about guideline implementation

- Practices typically cannot institute major changes in guidelines without approval by the health system's quality committee.

- Financial interests and input from specialists can shape health system guideline recommendations

Environmental Factors

Media and specialists can promote both evidence-based care and unnecessary care

- Patients increasingly hear about prevention through ads

- Specialists may promote services that do not follow guidelines

- For-profit organizations often promote unnecessary services

Traditional barriers to care were reported, but resources and solutions existed to help patients

- Clinicians and staff generally reported good access to care

- Health systems and communities try to reduce barriers to care

- Insurance coverage for prevention improves access to care, but lag time in coverage can delay adoption of new guidelines

\section{Guideline Factors}

Some guidelines are easier to adopt and implement

- If they can be incorporated into workflow

- If they can be programmed into the EHR as an alert

- If they can be appropriately measured for audit and feedback

- If patients who benefit from the services can be clearly identified

Some guidelines are harder to adopt and implement

- If they frequently change or change more radically

- If there are differing guidelines from multiple groups

- If the topic is less familiar to patients

- If the results can be difficult to explain to patients

- If they involve services outside the clinic
"Building the algorithm in the EHR is not just a guideline-based recommendation, it has to be a recommendation that the clinical organization agrees with from a financial or strategic perspective... does the screening have a negative financial impact on my organization? That's where the PSOs and CMOs and COOs decide." (Clinician)

"It all depends on the marketing of different things. . like for the new shingles vaccine, people are just coming to us like crazy." (Practice Leader)

"For colonoscopies, we got an arrangement with the local hospital medical group to take four to five uninsured patients per year to do colonoscopy" (Clinician)

"At a talk we had one time from an oncologist. . .he said he's been ordering [low dose chest CT for lung cancer screening] on all his patients now... he was completely unaware of the ages, the pack-year, nothing." (Clinician)

"They came out with the Prevnar recommendation, but Medicare didn't cover it for about a year. What good does it do if everybody's 65 and they don't have coverage?" (Clinician)

"Now that we have that built into our workflow to offer lung cancer screening to patients who qualify, that's been something we've gotten better about." (Clinician)

"I think having the risk calculators. . having some numbers to discuss with people about what we think their risk is and how much the risk might be reduced if they took medicine, I think that's helpful." (Clinician)

"AAA screens. . It's not one of our core quality measures... It's not a meaningful-use measure. . .I haven't ignored it. . .but I haven't directly addressed it because I feel like we're working on so many quality things." (Clinician)

"Mammography is a little harder...the guideline has changed so many times over the years." (Clinician)

EHR, electronic health record; PSO, patient safety organization; CMO, Chief Medical Officer; COO, Chief Operating Officer; CT, Computed Tomography.

ing implementation. Practices focused on streamlining workflow and defining roles while health systems maintained electronic health record (EHR) functionalities like alerts and quality measures. Interviewees reported that the media and specialists were the greatest environmental influences, promoting both evidence-based and at times excessive care. More traditional environmental factors, such as insurance coverage or transportation, were mentioned by interviewees but were less of a barrier to guideline-based care than media and specialists.

Interviewees noted that guidelines that are easier to measure, easier to explain, and involve more tangible health benefits are easier to adopt. Interviewees shared that guidelines frequently changing or differing between guidelines groups hinders adoption.
Thematic analysis revealed 3 categories describing how clinicians and practices decide which guidelines to adopt-clinician directed, practice directed, or health system directed (Table 2). Distinguishing factors included information learning and sharing, locus of decision control, communicating decisions, and primary implementation strategies. While most practices had a dominant approach, all used elements from each approach.

\section{Discussion}

Despite widespread agreement that adherence to clinical preventive guidelines improves health outcomes, timely implementation of new or changed guidelines in primary care continues to be suboptimal. Factors influencing guideline uptake are 
Table 2. Emerging Practice Categories for Approach to Guideline Adoption and Implementation

\begin{tabular}{|c|c|}
\hline Characteristics & Example Quotes \\
\hline \multicolumn{2}{|c|}{ Clinician-directed: Clinicians independently decide which guidelines to implement and how to implement them. } \\
\hline $\begin{array}{l}\text { Learning New Evidence: Clinicians learn on their own with } \\
\text { limited formal structures for clinicians or staff to learn from one } \\
\text { another. } \\
\text { Topic Leads: No identified clinician or staff leads to make } \\
\text { decisions for the practice. } \\
\text { Practice-Wide Updates: Information may come from the practice } \\
\text { or health system level, but there is no expectation for all } \\
\text { clinicians to abide by the same clinical guidelines. } \\
\text { Implementation Process: Done by individual clinicians. }\end{array}$ & $\begin{array}{l}\text { "I usually kind of decide that on my own based on what I've } \\
\text { learned up to this point in time and I'll search my own } \\
\text { experiences. I would be the first to say that at times I'm kind } \\
\text { of just a late adopter for certain things even though there may } \\
\text { be a guideline out. Sometimes I don't always agree with it" } \\
\text { (Clinician) }\end{array}$ \\
\hline \multicolumn{2}{|c|}{ Practice-directed: Practices collectively decide which guidelines to implement and how to implement them. } \\
\hline $\begin{array}{l}\text { Learning New Evidence: There are structures within the practice } \\
\text { through which clinicians can share knowledge with one } \\
\text { another. } \\
\text { Topic Leads: Individual clinicians or staff leads with topic } \\
\text { expertise lead the practice in a uniform clinical approach. } \\
\text { Practice-wide Updates: Information from the health system is } \\
\text { discussed among everyone in the practice, but ultimately the } \\
\text { practice has guideline decision-making autonomy. } \\
\text { Implementation Process: Large emphasis on workflow with } \\
\text { clinicians and staff working together to determine the best } \\
\text { workflow process. }\end{array}$ & $\begin{array}{l}\text { "We have two meetings a month within our clinic where } \\
\text { providers get together for just under an hour. One is a peer } \\
\text { review meeting. There's always education that's part of that. } \\
\text { And then we have a second meeting that's more focused on } \\
\text { workflows but oftentimes that dovetails with education and } \\
\text { being made aware of guidelines." (Clinician) } \\
\text { "The EHR, I mean we have these quality guidelines now that } \\
\text { kind of drive me insane. They're helpful to a point. They kind } \\
\text { of make me crazy too because I don't feel like those are as up- } \\
\text { to-date as we are maybe." (Practice Leader) }\end{array}$ \\
\hline \multicolumn{2}{|c|}{$\begin{array}{l}\text { Health system-directed: The health system decides which guidelines to implement and how to implement them and then informs practices and } \\
\text { clinicians. }\end{array}$} \\
\hline $\begin{array}{l}\text { Learning New Evidence: The health system regularly updates } \\
\text { clinicians and staff about new clinical guidelines. } \\
\text { Topic Leads: Clinicians and staff serve on health system } \\
\text { committees to make system level changes. } \\
\text { Practice-wide Updates: Evidence changes are reviewed by the } \\
\text { health system, often a quality committee, before making routine } \\
\text { changes to practice. }\end{array}$ & $\begin{array}{l}\text { "I chair our quality committee; so, if it's something that's really } \\
\text { new or different, then generally the quality committee will } \\
\text { take a look at it and we'll talk about, you know, is there } \\
\text { something we need to do? Should we advertise this? If it's } \\
\text { something really different from what the old guideline was, } \\
\text { usually the quality committee will talk about it and publicize } \\
\text { it." (Clinician) }\end{array}$ \\
\hline $\begin{array}{l}\text { Implementation Process: The EHR is utilized heavily to define } \\
\text { the workflow. }\end{array}$ & $\begin{array}{l}\text { "We just made it happen. It wasn't open for discussion to be } \\
\text { honest. There's not a lot open for discussion as far as changes } \\
\text { go. We just say this is how it's going to be; just grumble and } \\
\text { move forward." (Practice Leader) } \\
\text { "we do have a lot of standing orders and we do have a lot of } \\
\text { adult immunizations, things that the nurses can do on their } \\
\text { own..." (Practice Leader) }\end{array}$ \\
\hline
\end{tabular}

EHR, electronic health record.

complex and involve an interplay of patient, clinician, practice, health system, environment, and guideline factors. Improving guideline uptake requires interventions that promote synchrony of all factors.

A common barrier to guideline uptake for clinicians and patients was fears and worries. This barrier was particularly difficult because it impacted care if either the clinician or patient had fears or worries. This dynamic was further aggravated by environmental factors like media and specialists, which may have financial gains and maybe biased to promoting overuse.

By examining the interplay of patient, clinician, and environmental factors, one can consider interventions beyond those that address individual factors. For instance, enhancing the patient-clinician relationship may improve guideline adoption and diminish adverse influences of media and specialists. Further ensuring that guideline developers are not influenced by financial gain and that guidelines include cost effectiveness information would add to uptake.

The categories for how practices adopt and implement guidelines shown in Table 2 provide important insights into the unique barriers and needed interventions to overcome barriers for different settings. In some practices, clinicians have more autonomy to implement changes while in others require health system leadership approval. Practices with greater clinician autonomy are at risk for greater variation in care. Interventions focusing on clinician education may be most helpful. Practices 
with stronger practice or health system decision making may struggle with delays in adopting changes due to time required for leadership buy-in or for electronic health record changes. Further, financial incentives and specialist needs may make it more difficult for systems to implement the right primary care approach. Interventions may need to streamline approval and support primary care needs. In all cases, advancing the nondominant adoption and implementation style may also be beneficial. ${ }^{11}$

\section{Limitations}

All participating practices were part of a practice-based research network and may be more proactive in keeping up to date with guidelines. In addition, no patients were interviewed and identified patient factors are limited to clinician and practice leader observations.

\section{Conclusion}

Multiple levels of factors influence guideline uptake and need to be considered within the context of each practice setting to improve adoption and implementation of evidence-based guidelines. Categorizing practices as clinician-directed, practice-directed, or health system-directed may help identify optimal implementation strategies for different practice settings.

The authors thank Virginia Ambulatory Care Outcomes Research Network and Oregon Community Health Information Network for their support and coordination of activities. We thank Paulette Kashiri, Nate Warren, and Erik Geissal for recruiting participants and supporting practice activities.

To see this article online, please go to: http://jabfm.org/content/ 33/2/271.full.

\section{References}

1. Graham R, Mancher M, Wolman DM, Greenfield S, Steinberg E, eds. Clinical practice guidelines we can trust. Washington, DC: National Academies Press (US); 2011.

2. Lee G, Carr W. Updated framework for development of evidence-based recommendations by the Advisory Committee on Immunization Practices. MMWR Morb Mortal Wkly Rep 2018;67:1271-2.

3. McGlynn EA, Asch SM, Adams J, et al. The quality of health care delivered to adults in the United States. N Engl J Med 2003;348:2635-45.

4. Haas JS, Sprague BL, Klabunde CN, et al. Provider attitudes and screening practices following changes in breast and cervical cancer screening guidelines. J Gen Intern Med 2016;31:52-9.

5. Raz DJ, Wu GX, Consunji M, et al. Perceptions and utilization of lung cancer screening among primary care physicians. J Thorac Oncol 2016;11: 1856-62.

6. Magnani CJ, Li K, Seto T, et al. PSA testing use and prostate cancer diagnostic stage after the 2012 U.S. Preventive Services Task Force guideline changes. J Natl Compr Cancer Netw 2019;17:795-803.

7. Schuchat A, Anderson LJ, Rodewald LE, et al. Progress in vaccine-preventable and respiratory infectious diseases-First 10 years of the CDC National Center for Immunization and Respiratory, 2006-2015. Emerg Infect Dis 2018;24: $1178-87$.

8. Turner S, D'Lima D, Hudson E, et al. Evidence use in decision-making on introducing innovations: A systematic scoping review with stakeholder feedback. Implement Sci 2017;12:1-12.

9. Francke AL, Smit MC, De Veer AJE, Mistiaen P. Factors influencing the implementation of clinical guidelines for health care professionals: A systematic meta-review. BMC Med Inform Decis Mak 2008;8:1-11.

10. Kovacs E, Strobl R, Phillips A, et al. Systematic review and meta-analysis of the effectiveness of implementation strategies for non-communicable disease guidelines in primary health care. J Gen Intern Med 2018;1-13.

11. Gagliardi AR, Alhabib S. Trends in guideline implementation: a scoping systematic review. Implement Sci 2015;10:54.

12. USPSTF. Screening for breast cancer: U.S. Preventive Services Task Force. Ann Intern Med 2016;164:279-96.

13. USPSTF. Screening for prostate cancer: U.S. Preventive Services Task Force Recommendation Statement. Ann Intern Med 2012;157:120-34.

14. USPSTF. Screening for cervical cancer: U.S. Preventive Services Task Force Recommendation Statement. Ann Intern Med 2012;156:880-91.

15. USPSTF. Screening for lung cancer: U.S. Preventive Services Task Force Recommendation Statement. Ann Intern Med 2014;160:330-8.

16. Kobayashi $M$, Bennett NM, Gierke R, Almendares $\mathrm{O}$, Moore MR. Intervals between PCV13 and PPSV23 vaccines: recommendations of the Advisory Committee on Immunization Practices (ACIP). MMWR Morb Mortal Wkly Rep 2015;64:944-8.

17. Fiore AE, Uyeki TM, Broder K, et al. Prevention and control of influenza with vaccines: recommendations of the Advisory Committee on Immunization Practices (ACIP), 2010. MMWR 2010;59:1-61.

18. USPSTF. Statin use for the primary prevention of cardiovascular disease in adults: U.S. Preventive 
Services Task Force Recommendation Statement. JAMA 2016;316:1997-2007.

19. USPSTF. Screening for high blood pressure in adults: U.S. Preventive Services Task Force Recommendation Statement. Ann Intern Med 2015;163:778-86.
20. Crabtree BMW. Doing qualitative research. Thousand Oaks, CA: Sage Publications, Inc; 1999.

21. Miles MB, Huberman AM. Qualitative data analysis. An Expanded Sourcebook. 2nd ed. Thousand Oaks, CA: Sage Publications, Inc; 1994. 


\section{Appendix}

\section{Interview Guide}

Interviewer to inform participant that interview will be recorded and not to mention identifying info.

Purpose: to understand factors that come into play when implementing clinical practice guideline changes. For purposes of the study, we focused on cervical cancer, breast cancer, lung cancer, prostate cancer, hypertension, hyperlipidemia, pneumonia vaccine and influenza vaccine. However, we will be focusing broadly on how you implement clinical practice guidelines in your practice.

Questions

1. Learning about new practice guidelines

a. How do you generally keep up-to-date with changes in evidence?

i. What specific tools, publications do you use?

ii. How do you decide what things are relevant to your practice?

b. What is your experience with implementing new guidelines that involve:

i. New services being offered to patients? (e.g. Flu vaccines to all adults rather than just those at high risk, Prevnar vaccines to all individuals $>65$ yo)

ii. Making a service less frequent? (e.g. Increasing interval between pap smears, mammograms)

iii. Changes in thinking about when to offer a service? (e.g. Statin recommendations based on cardiovascular risk rather than specific cholesterol lab values)

2. Patient factors

a. How do you talk to your patients about new guidelines?

i. Resources?

ii. Handouts?

iii. Belief systems?

iv. Family members?

b. Experiences when patients ask you about new guidelines?

3. Community/socioeconomic factors (Environmental)

a. Describe your patient population.

b. Experiences with your patients having problems accessing recommended clinical services.

i. Transportation

ii. Insurance coverage/co-payments

iii. Time off work

iv. Ease of scheduling/wait times/available times (after hours, etc)

c. Are there community organizations or groups that help with recommended clinical services? Specialists?

4. Clinic and system support for implementation

a. Describe your practice setting and relationship with a health system.

i. Group/solo

ii. Interactions with colleagues

iii. Support systems (RN, MA, SW, psych, admin?)

b. How does your clinic support your implementation of new guidelines?

i. EHR support? ii. Nursing/medical assistant support?

iii. Automated reminders?

iv. Letters?

v. Patient resources provided?

c. How does your health system support your implementation of new guidelines? (same prompts)

5. Guideline specific

a. Are there guidelines that you have find easier/ harder to implement than others? Why? (Review one-page handout)

i. Cultural context and beliefs?

ii. Media?

iii. Which guideline do you follow? Any unique issues?

Interviewer will ask if any other questions or comments. Interviewer to thank clinician for participation.

\section{Examples of Changes in Clinical Guideline over the past 10 years}

Breast Cancer Screening

- Women age 40-49 yrs: Change from routine screening to personalizing age to start mammogram screening

- Women age 50-75 yrs: Change from annual to biennial screening

Cervical Cancer Screening

- Change to delay screening age from age of first sexual intercourse to 21 yo

- Change to extend screening interval from every 1 year to every 3 years

- New option for co-testing for HPV for ages 30-65

Prostate Cancer Screening

- New recommendation against routine PSA screening.

Lung Cancer Screening

- New recommendation to obtain annual CT chest for all individuals age 55-80 with a 30 pack-year history unless they have not smoked in the last 15 years

Pneumonia (Prevnar 13) Vaccination

- New recommendation to give PCV-13 vaccine to all individuals age 65 and older

Flu Vaccination

- Change to expand annual flu vaccines from only those at risk under age 65 to all patients after 6 months of age.

Blood Pressure Goals

- Change in goal from 120/80 to $140 / 90$ for everyone and 150/90 for everyone age 60 or greater.

Statin Recommendations

- Change from titrating statin to specific cholesterol levels to giving statins based on cardiovascular risk calculations

- Change to only using statins

- May not need to check cholesterol levels if on appropriate statin 